\title{
Use of SVD decomposition to increase signal and noise ratio on THz imaging measurements.
}

\author{
by C. Pradere*, J.P. Caumes**, E. Palomo* and J.C. Batsale*
}

* Univ. Bordeaux, I2M, UMR 5295, F-33400 Talence, France, c.pradere@i2m.u-bordeaux1.fr, e.palomo@i2m.u-bordeaux1.fr and jc.batsale@i2m.u-bordeaux1.fr

${ }^{*}$ NeTHIS New THz Imaging System, Bordeaux Technowest, Bureau 7, 25, rue Marcel Issartier, 33702 , Mérignac Cedex, jean-pascal.caumes@nethis-thz.com

\begin{abstract}
In this paper a processing method based on Singular Value Decomposition is proposed to measure $\mathrm{THz}$ images by using a coupled system of Tera Thermal Converter and IR thermography. By using such method the Signal to Noise Ratio could be increased from a factor 50 and the acquisition rate decreased by 10 times. Based on an analytical thermal modelization of the tera thermal conversion, several parameters will be pointed out in order to realize a good quality image in reasonable time with such $\mathrm{THz}$ camera. To illustrate this purpose, the use of SVD and proposed acquisition method leads to $\mathrm{THz}$ image of a $5 \mathrm{~cm}$ diameter object in $100 \mathrm{~ms}$.
\end{abstract}

\section{Introduction}

Terahertz $(\mathrm{THz})$ imaging constitutes a promising technology for many large tomography applications in many domains. The improvement of $\mathrm{THz}$ instrumentations since last years is motivated in part by the large range of applications as [1-4]: imaging, sensing, spectroscopy, medicine, art conservative studies and non-destructive testing... Up to now, optical radiometry of $\mathrm{THz}$ detectors has been missing in the $\mathrm{THz}$ range. In fact, most of the published $\mathrm{THz}$ images were performed by using a scanning system with a single pixel detector [5, 6]. Although absolute traceable $\mathrm{THz}$ power measurements have recently been investigated [7-9]. Nowadays, this technique is being substituted by $\mathrm{THz}$ imaging based on array of sensors. Even if, several technologies are in competition, an efficient method of determining $\mathrm{THz}$ detector characteristics is needed. Nevertheless, it is already difficult to find a complete and powerful system which is high sensitive, broadband and with high frame rate acquisition. In fact, the performances of $\mathrm{THz}$ detector are measured as function of their noise limitation represented by the Noise Equivalent Power (NEP). The NEP corresponds to the minimum signal power measurable by the detector (or pixel in the case of imaging) in order to obtain a signal to noise ratio (SNR) of one.

Recently, a THz camera based on an uncooled microbolometer $160 \times 120$ pixels array with pitch of $52 \mu \mathrm{m}[10]$ has been developed at INO and first transmission and reflection images showed a promise device. Measurements in the $\mathrm{THz}$ region has been performed using non-uniform low-power quantum-cascade laser (QCL) and uniform high-power far-infrared laser (FIR laser) beams at 2.54, 3 and $4.25 \mathrm{THz}$. Another high improvement was performed by [11] where they have developed highly sensitive microbolometers with a specific design for $\mathrm{THz}$ detection based on an innovative use of antennas and resonant cavity, resulting in sensitivity improvement and frequency agility. They demonstrated the possibility to image low power signals of beam spots focused by mean of different optics.

In this study we will show that using a quantum infrared camera coupled with a Tera Thermal Converter is also a robust and easy to use concept $[12,13]$ in order to perform high sensitive and fast 2D broadband far infrared imaging. The main advantage of this system is to use a deported bolometer allowing to manage the multiscale change induce by the broadband wavelength. One drawback is the sensitivity induces by the thermal conversion. The main objective of this paper is to establish that these cost-effective imaging systems can be clearly optimized using thermal methods to gain acquisition rate, sensitivity or physical parameters extractions. These analytical or numerical methods have been clearly experienced in different physics [14-16] and have permitted gain on different thermal systems. Coupling high-level thermal analysis with optical technics leads to a new generation of far infrared broadband bolometer. Moreover, as they are extrapolated from the infrared technology, they benefit from the well-mastered infrared manufacturing process and from all the read-out procedures developed for thermal imaging.

\section{Thermal analytical analysis of the Tera Thermal Converter (TTC)}

\subsection{Thermal modelization of the conversion}

The TTC usually used for the thermal conversion is a very thin and homogeneous square carbon sheet of $4 \mathrm{~cm}$ by $4 \mathrm{~cm}$ with a thickness of $15 \mu \mathrm{m}$. By using classical thermal characterization $[17,18]$ the thermal diffusivity of the TTC has been estimated and the obtained values in each direction are equal and close to 4.16.10-7 $\mathrm{m}^{2} \cdot \mathrm{s}^{-1}$. So, from a thermal point of view, it is assuming that the TTC is a thin homogeneous media with internal heat source due to the incident $\mathrm{THz}$ radiation. Due to the very large ratio between length and thickness it can be verified that the temperature is constant overall the thickness of the TTC (ie in z direction). Moreover, as the absorption of the incident $\mathrm{THz}$ radiation is a function of the thickness (Beer Lambert law), we assumed the 
heat source is constant over $\mathrm{z}$ direction due to the large ratio between the wavelength (around $3 \mathrm{~mm}$ and the TTC thickness $15 \mu \mathrm{m}$ ). Finally, the thermal behavior of the TTC is represented by a 2D thermal model where the heat source due to $\mathrm{THz}$ radiation is represented as an initial temperature with adiabatic boundaries conditions. This basic thermal model is solved [18] in the Fourier space with the following expression:

$$
\theta\left(\alpha_{n}, \beta_{m}, t\right)=\theta\left(\alpha_{n}, \beta_{m}, 0\right) \exp \left[\left(-a\left(\alpha_{n}^{2}+\beta_{n}^{2}\right)+H\right) t\right]
$$

Where $\theta\left(\alpha_{n}, \beta_{n}, t\right)(\mathrm{K})$ is the temperature in the Fourier space, $\alpha_{n}=n \pi / L x$ and $\beta_{m}=m \pi / L y\left(\mathrm{~m}^{-1}\right)$ are the eigenvalues, $a$ is the thermal diffusivity $\left(\mathrm{m}^{2} \cdot \mathrm{s}^{-1}\right), \mathrm{H}$ is the coefficient of characteristic time due to heat losses $\left(\mathrm{s}^{-1}\right)$ and $\theta\left(\alpha_{n}, \beta_{n}, 0\right)(\mathrm{K})$ is the initial value of the temperature relative to the incident $\mathrm{THz}$ heat source. By using an inverse Fourier integral transform and a convolution (with a time function) of the impulse thermal response of Eq. (1), the temperature field $T(x, y, t)$ can be calculated. As an example, the results obtained with the analytical solution (Eq. 1.) calculated for a step function and with the following parameters: thermal diffusivity of $10^{-7} \mathrm{~m}^{2} \cdot \mathrm{s}^{-1}$ close to the one of the TTC, $\mathrm{H}$ equal to $0.1 \mathrm{~s}^{-1}, \mathrm{Lx}=\mathrm{Ly}=4 \mathrm{~cm}$ and an initial temperature equal to $1^{\circ} \mathrm{C}$ with a centered square shape are represented figure 1.
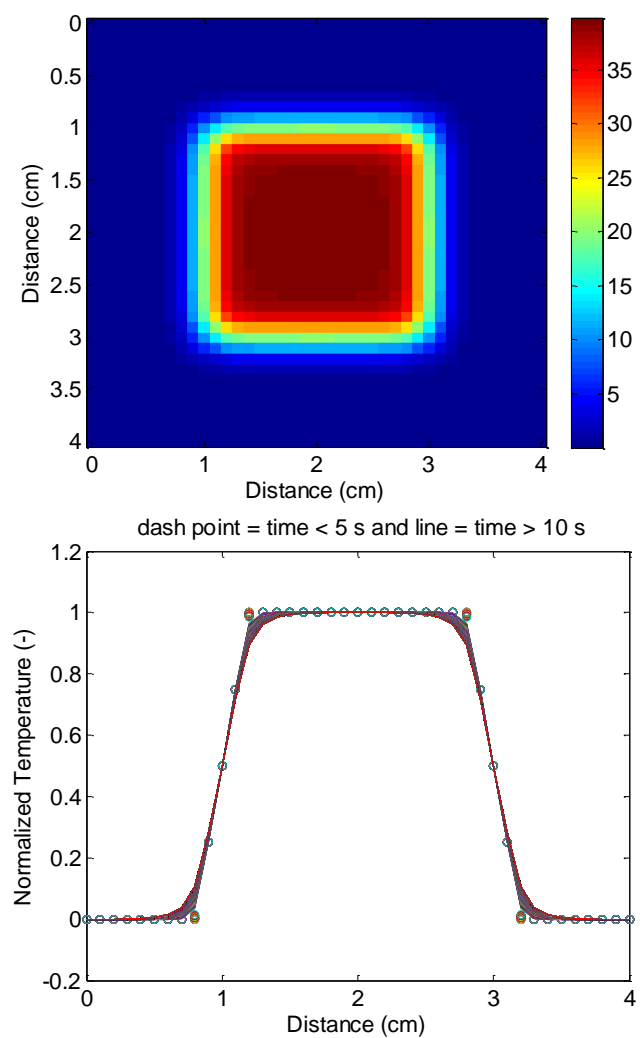

(a)

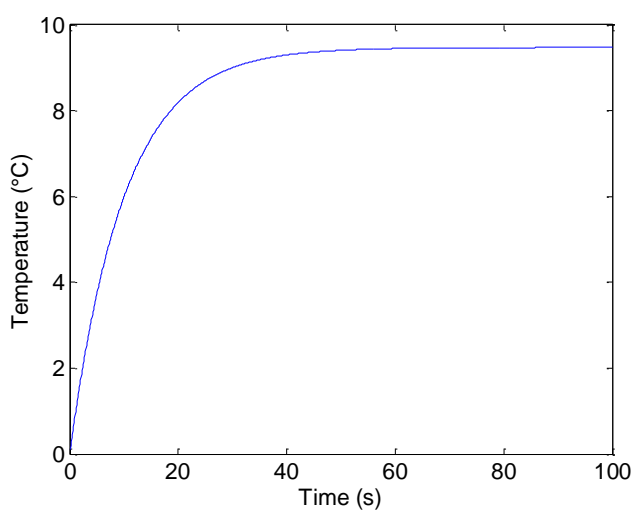

(b)

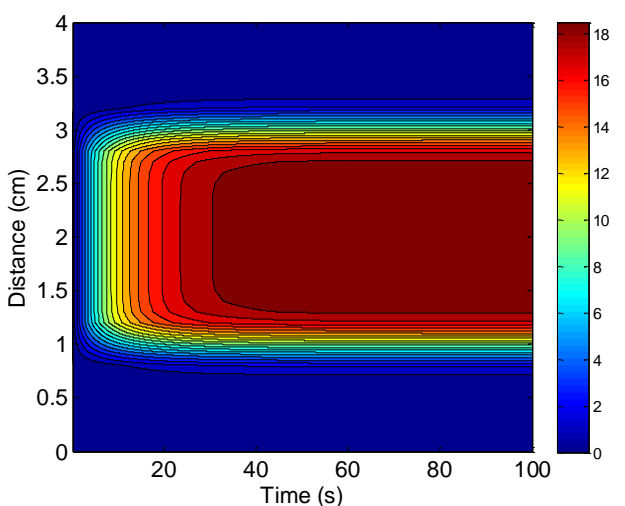

Fig. 1. Results of the analytical calculation of the heat transfer on the TTC: a), the temperature field at steady state is represented, b) the average temperature overall the space is plotted as function of time, c) the normalized temperature profile in $x$ direction as function of time and $d$ ), the field of temperature profile (averaged in $y$ direction) as function of $x$ direction and time.

First, figure 1.b the time to reach the steady state is long around $50 \mathrm{~s}$. Moreover, figure 1.a when the steady state is reached the temperature field is "blurred" due to the in plane thermal diffusion of the media. As a consequence, it is very important to work at short time to avoid diffusion problem in order to have a temperature profile in good agreement with the true shape of the incident source. From a thermal point of view and with the knowledge of the thermal properties an inverse method could be used to retrieve the initial value of the temperature. Nevertheless, as shown figures 1.c and 1.d we can notice that at short time $t<5 \mathrm{~s}$ the shape of the temperature profile is constant with an increasing amplitude. During this time, the diffusion effect is week and corresponds to spatial variation less than one pixel of the initial shape of the source (here $250 \mu \mathrm{m}$ ). Finally, to perform accurate measurement in THz domain by using such TTC it is very important to work at short time where the diffusion due to thermal heat transfer is minor and where it is not necessary to performed inverse method to estimate the heat source (ie retrieve the initial temperature). At this stage, two approaches can be considered. The first one is analytical and based on the Fourier integral transform of the images versus the space variables $x$ and y. In such a « physical » approach, the estimation of the thermal diffusivity of the sample is absolutely necessary introducing uncertainties and heavy computations and data manipulations.

The second approach will consist, without any knowledge about the physical mechanisms, in implementing a Singular Value Decomposition (SVD) of the entire temperature data related to the transient field 
evolution. The first advantage is that the truncation of the decomposition reduces considerably the amount of useful data (from about 300 millions of data to about 3000 data). The second advantage is to filter the signal without any knowledge about the thermal diffusivity.

\subsection{Validation on noisy analytical solution by using SVD as thermal processing}

As depicted in the thermal modelization part, the problem is the estimation of the initial temperature field directly linked to the shape of the heat source (or THz radiation optically projected to the TTC). During the times which are lower to the thermal characteristic diffusion time of one pixel (around $1.5 \mathrm{~s}$ for a pixel of $250 \mu \mathrm{m}$ with thermal diffusivity of $4.16 .10^{-7} \mathrm{~m}^{2} \cdot \mathrm{s}^{-1}$ ), the shape of the incident source (or $\mathrm{THz}$ radiation) is quite constant (because diffusion effect occurred in less than one pixel of the measured temperature) and the temperature resulting from the $\mathrm{THz}$ radiation source increases. In that case, the thermal problem is reduced to the research of an initial temperature field which is directly proportional to the heat source (incident $\mathrm{THz}$ radiation). In order to avoid a complete knowledge of the thermal properties it has been demonstrated that method based on singular value decomposition are great suitable. From years, singular value decomposition (SVD), PCA/KLD/SVD is a very common tool today in image processing and signal processing problems for compression and noise reduction [14]. It is also widely used for signals classification, data clustering and information retrieval problems [15-16]. In thermal analysis, SVD-based methods have been developed for efficient reduction of linear and non linear heat transfer problems [19-20], as well as for solving heat transfer inverse problems dealing with unknown heat sources [21-22]. From a mathematical point of view, applying a SVD on a thermal field of temperature results in the following formulation:

$$
T(x, y, t)=\sum_{m=1}^{r_{0}} U_{m}(x, y) S_{m} V_{m}(t)
$$

The properties of this decomposition are important. First, the matrix $U$ and $V$ are orthogonal, the space $(x, y)$ and time $(\mathrm{t})$ are separated, the eigenvalues given by the diagonal of the matrix $\mathrm{S}$ are ranging in decreasing exponential mode. In this work, only the properties of optimal filtering of this decomposition will be used. For that, the sum of the decomposition will be truncated with a value of $\mathrm{r} 0<6$ where the eigenvalues are always greater than the variance of the noise. Then, it becomes possible to obtain an initial field of temperature.

In fact, figure 2, the SVD decomposition is applied and the temperature field is reconstructing at several times of the analytical solution corresponding to the thermal response of the TTC to a step function during $1 \mathrm{~s}$. First, figure 2.a, the raw temperature field is compared to the processing one. Here, the time step between each image is equal to $100 \mathrm{~ms}$ with a starting point at to $=100 \mathrm{~ms}$. After $300 \mathrm{~ms}$ (image number 3 figure 2.a) the shape of the square could be clearly seen in the case of the SVD whereas on raw data it is quite impossible to distinguish the source. Further, by the knowledge of the analytical solution and the level of noise it is possible to calculate the SNR figure 2.b and the gain of the SVD methods figure 2.d in comparison with raw data. Then figure 2.c the shape of the square in $x$ direction is compared to the original one for both raw and processing data. It could be show that even for the first image $(t=100 \mathrm{~ms})$ the shape of the square is well evaluated with SVD whereas the noise is huge for raw data. As a consequence, figure 2.d, the gain produce by the SVD is very high at short time where the signal is very low comparing to the noise. Whereas, Fig 2.b, at long time (here $500 \mathrm{~ms}$ ) the gain of the SVD is stable and constant comparing to raw data SNR which increases.

To conclude this analytical part, the main interesting point is that the use of SVD on thermal conversion increases the SNR ratio at short time allowing very fast imaging of good quality.

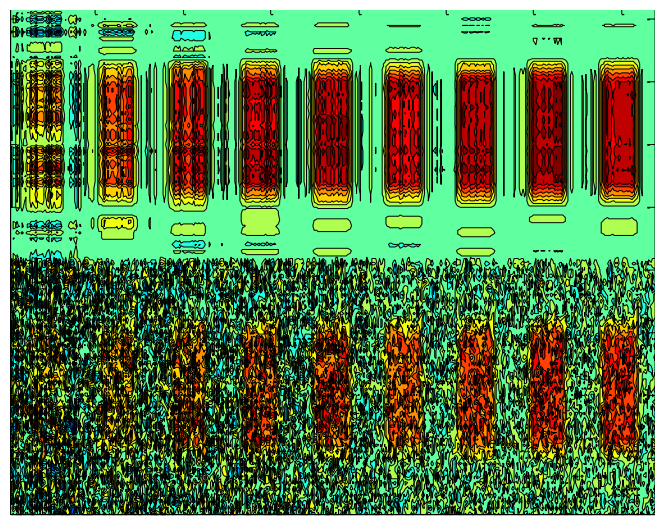

(a)

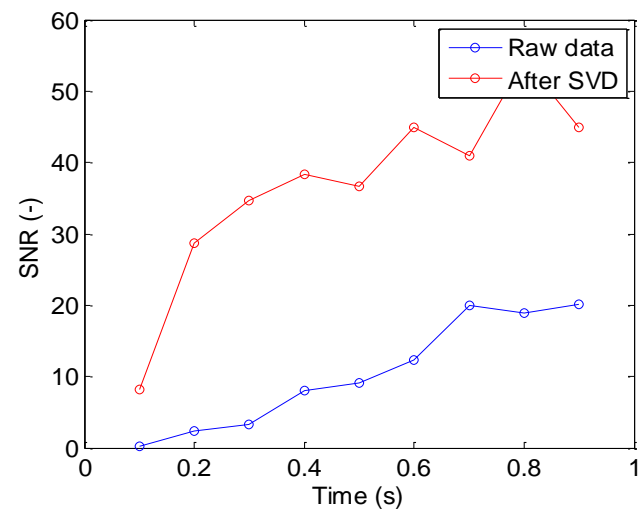



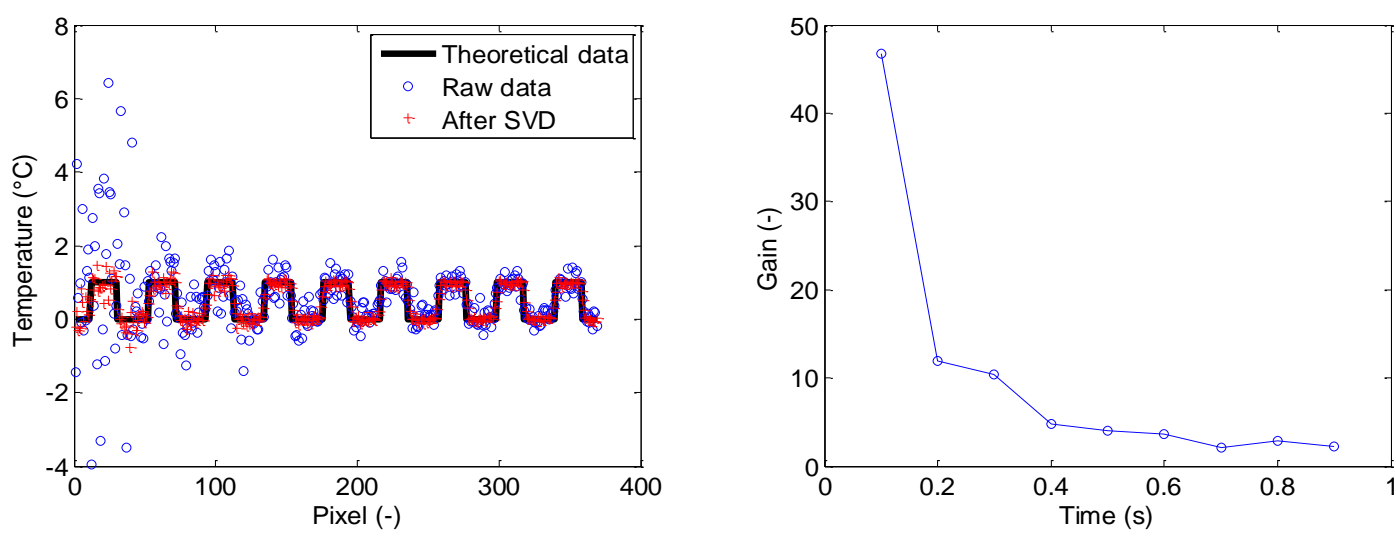

(c)

Fig. 2. a), Temperature field for raw noisy data (bottom) and processing data with SVD (top) as function of time (time step of 100 ms between two images), b) SNR ratio calculated as function of time for raw and processing data, c), extracted shape of the square along $x$ direction for each time and d), gain calculate as the ratio of SNR between raw and SVD data as function of the acquisition time.

\section{Experimental results}

\subsection{Description of the set-up}

In figure 3, photography of the device is presented. First, a millimetric Gunn diode of $110 \mathrm{GHz}$ (wavelength of $2.8 \mathrm{~mm}$ ) from Virginia Diodes is use as source. The power of this diode is $40 \mathrm{~mW}$. Then b) a mechanical shutter from Uniblitz is used to modulate the continuous beam. To perform an image of any object, a $\mathrm{THz}$ objective design by ALPhANOV is used with a magnitude of 1 . Then to convert the incident optical radiation in the IR domain, an objective (d) from NeTHIS is associated with a FLIR SC7000 IR camera (e). The IR camera is a MCT sensor with a broad range of 7 to $14 \mu \mathrm{m}$ and a sensor array of $256 \times 320$ pixels.

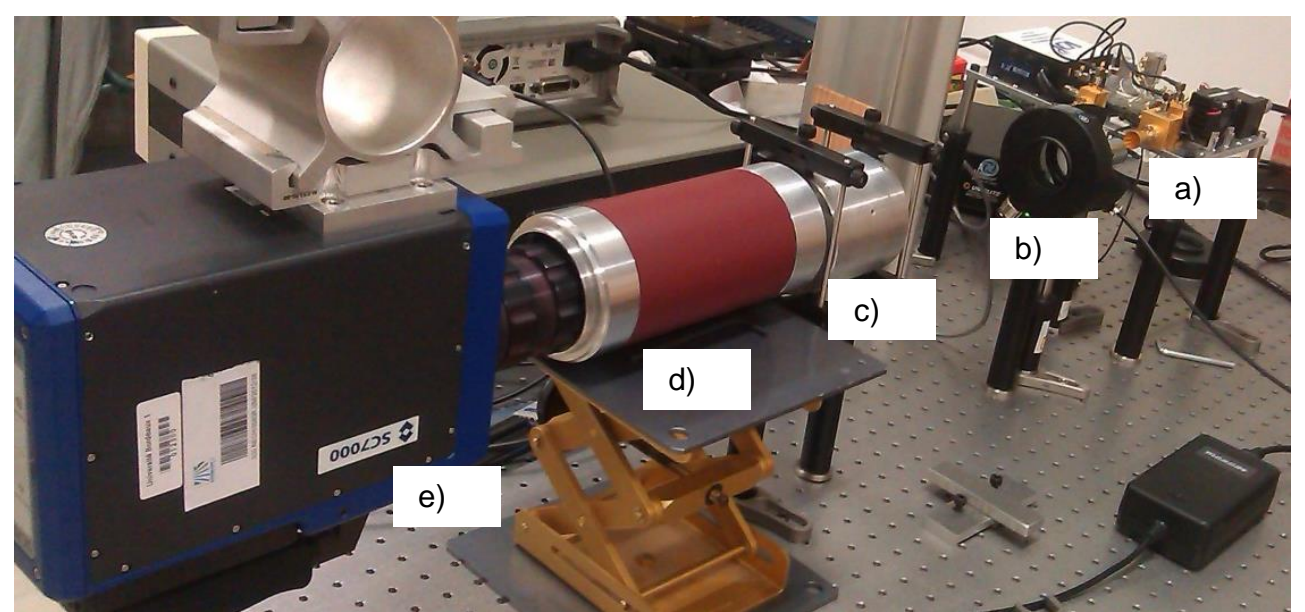

Fig. 3. Photography of the set-up: a), Gunn diode of $110 \mathrm{GHz}$, b) mechanical shutter, c) $\mathrm{THz}$ objective of magnitude 1, d), THz-IR objective with Tera Thermal Converter and e) IR camera.

\subsection{Measurement procedure and explanation of IT}

The main idea of such far infrared camera (figure 4) measurement procedure is to create a new $\mathrm{THz}$ integration time (IT). In fact, SC 7000 IR camera used a snapshot mode. In that case, both the IT and acquisition rate can be controlled with values going from $1 \mu \mathrm{s}$ to $1 \mathrm{~ms}$ for the IR-IT and $1 \mathrm{~Hz}$ to $1 \mathrm{kHz}$ for the frequency range. The principle is to synchronize the modulated $\mathrm{THz}$ signal (coming from pulsed $\mathrm{THz}$ beam or for continuous $\mathrm{THz}$ sources by using mechanical chopper or shutter) with the acquisition signal of the IR camera.

By doing this, a new THz frequency is creating going from 0.1 to $1000 \mathrm{~Hz}$. From these considerations, the TTC thermal response is recording during the rising parts of the THz excitation. Finally, by using data processing method based on singular value decomposition only one resulting image at the frequency given by the modulation of the THz source is extracted. As depicted in the thermal modelization part, the THz-IT should never be higher that the thermal diffusion characteristic time (usually around $1 \mathrm{~s}$ ) given by the thermal properties of the TTC and the pixel size of the IR image. 


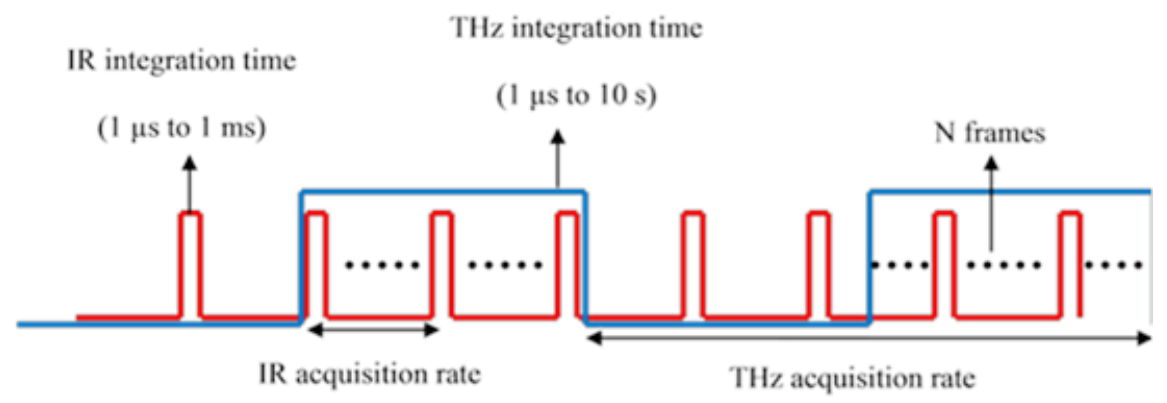

( $1 \mathrm{~Hz}$ to $\mathrm{I} \mathrm{kHz}$ )

$(0.1 \mathrm{~Hz}$ to $1 \mathrm{kHz})$

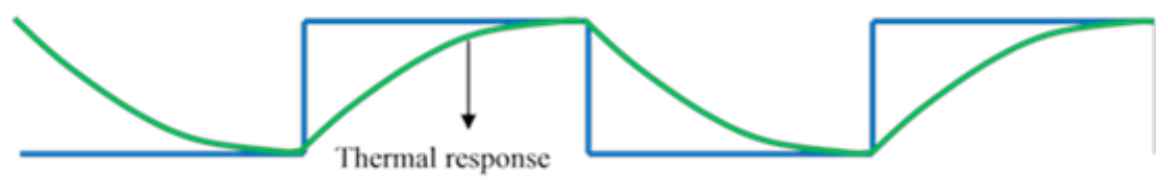

of the TTC

Fig. 4. Schema of the acquisition method: at the top, explanation of the THZ Integration Time (THZ-IT), the InfraRed Integration Time (IR-IT) and both frequency acquisition and at the bottom, example of the Tera Thermal Converter (TTC) thermal response as function of the THz acquisition rate.

\section{3 $\mathrm{THz}$ beam characterization}

By using the set-up and the measurement method describe before, a first experimentation was performed for the measurement of the THz diode shape. The THZ-IT was set to $1 \mathrm{~s}$ the IR-IT to $600 \mu \mathrm{s}$ and the frequency acquisition of the camera at $100 \mathrm{~Hz}$. According to the Eq. (2), the SVD decomposition is applied for several time step going from $60 \mathrm{~ms}$ to $760 \mathrm{~ms}$ by step of $100 \mathrm{~ms}$. The obtained results are reported figure 5 where the raw data are compared to the processing one by using SVD and $r_{0}=3$.

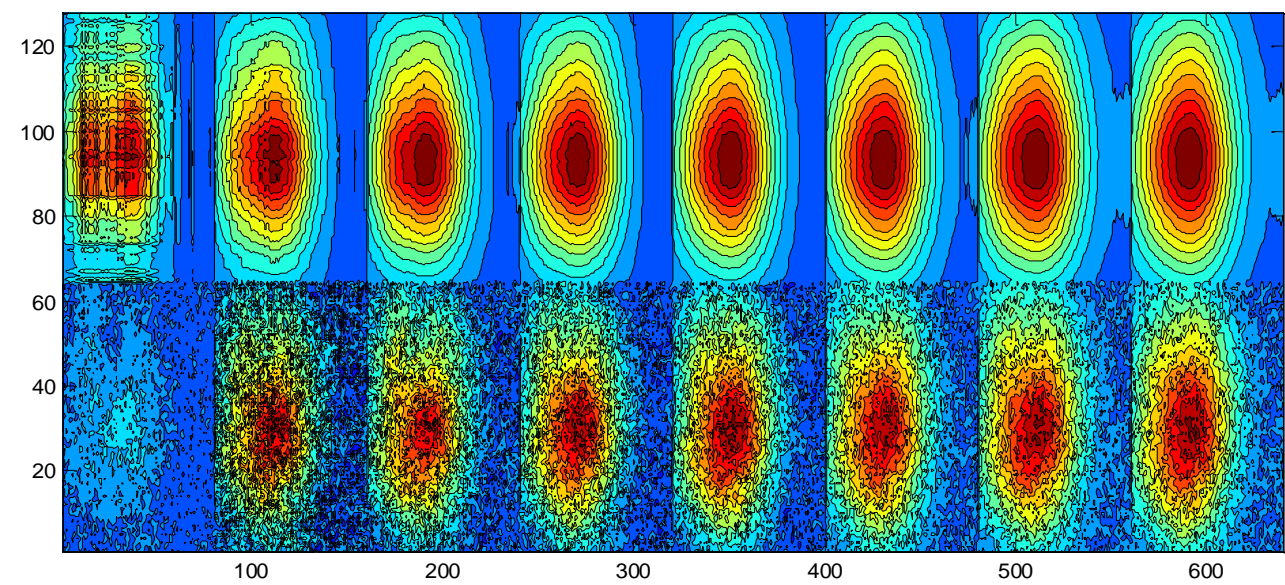

Fig. 5. Measured $\mathrm{THz}$ beam at $110 \mathrm{GHz}$ with $20 \mathrm{~mW}$ power as function of the acquisition time from $60 \mathrm{~ms}$ to 760 $\mathrm{ms}$ by step of $100 \mathrm{~ms}$ and the following parameters, camera acquisition rate is $100 \mathrm{~Hz}, \mathrm{THZ}-\mathrm{IT}$ is $1 \mathrm{~s}$ and IR-IT is $600 \mu \mathrm{s}$ : at the top, data processing by SVD decomposition with rO = 3 and at the bottom, raw data.

First, it is important to notice that even after $760 \mathrm{~ms}$ the raw data are very noisy making difficulties to clearly identify the shape of the THz beam. On the other side, by using SVD even after $160 \mathrm{~ms}$ the image of the beam has good quality with low noise. By this example, the interest of SVD in image measurement in THz domain by using a TTC and IR camera is illustrated. By this way, the image quality are improved and the time of acquisition also. Depending on the power of the beam, the time to make an image could attain 1 ms comparing to $1 \mathrm{~s}$ or more for raw data. Moreover, earlier the image is taken better is the sharp of the image. Depending on the optical transparency of any object regarding to the $\mathrm{THz}$ wavelength, these parameters (THZ-IT, frequency acquisition, number of eigenvalues (ro) of the SVD....) should be adjusted for a good quality of the measurement. 


\subsection{THz imaging of metallic object inside cardboard box}

A last example concerning the image of a metallic object inside a cardboard box was made. The results and the parameters of the measurements are depicted figure 6 .

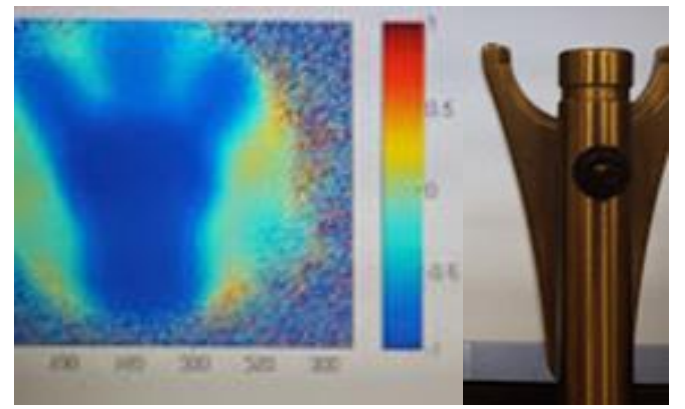

Fig. 6. THz imaging of a metallic object placed inside a cardboard box. The size of the image is $5 \mathrm{~cm}$ of diameter (size of the incident beam and TTC), the THz Integration time is $100 \mathrm{~ms}$ and the image is processing by $S V D$ with a $\mathrm{rO}=3$ : left part, is the resulting $\mathrm{THz}$ image measured with the device and right, picture of the object in visible.

\section{Conclusion and Further works}

To summarize the main results, depending on the thermal properties of the converter (TTC), the maximum integration time of the $\mathrm{THz}$ imaging should be adjusted. This time is strongly linked to the spatial resolution of the image and the thermal diffusivity of the converter. Here, the comparison between an analytical solution and measured one display a maximum time close to $1 \mathrm{~s}$. This time is totally independent of the processing method and influence directly on the quality of the image in term of blurring problem. In fact, due to thermal diffusion on the converter, if the integration time is taken higher than this limit the diffusion process (due to heat transfer) will blur the image.

The second important point is the signal to noise ratio strongly dependant of the power of the source, the thermal conversion factor and the camera sensitivity. In that case, whatever these parameters are, the use of SVD will hugely increase this ratio as well as drastically decreasing the acquisition time.

To conclude in this paper, the interest to include signal processing as SVD directly in the acquisition system could be a robust method to decrease the effect of noise on raw measured data and to increase the acquisition rate. From this point of view and with such device, $\mathrm{THz}$ tomography could be envisioning in reasonable time and good sensitivity.

\section{REFERENCES}

[1] Mittleman D., [Sensing with Terahertz Radiation], Springer Series in Optical Sciences, New York, Vol. 85, 337 p. (2003).

[2] Chan W. L., Deibel J. and Mittleman D. M., “Imaging with terahertz radiation”, Rep. Prog. Phys. 70, 1325 (2007).

[3] Redo-Sanchez A. and Zhang X.-C., "Terahertz science and technology trends", IEEE J. Sel. Top. Quantum Electron. 14, 260 (2008).

[4] Jansen C., Wietzke S., Peters O., Scheller M., Vieweg N., Salhi M., Krumbholz N., Jördens C., Hochrein T. and Koch M.,"Terahertz imaging: applications and perspectives", Appl. Optics 49, E48 (2010).

[5] B. B. Hu, M. C. Nuss, and B. B. Hu, "Imaging with terahertz waves," Opt. Lett. 20(16), 1716-1718 (1995).

[6] P. U. Jepsen, D. G. Cooke, and M. Koch, "Terahertz spectroscopy and imaging - modern techniques and applications," Laser \& Photon. Rev. 5(1), 124-166 (2011).

[7] C. Pradere, J. P. Caumes, J. Toutain, E. Abraham, B. Chassagne, J.C. Batsale (2013), "Absolute selfcalibrated room-temperature terahertz powermeter". Applied Optics, ISSN: 0003-6935, 52(11), 23202324.

[8] Wegner L., Hübers H. W., Meindl P., Richter H. and Steiger A., "Towards traceable radiometry in the terahertz region",Metrologia 46, S160 (2009).

[9] Steiger A., "Traceable terahertz power measurement by using optical methods", Proc. SPIE 7485 74850J (2009).

[10] Bolduc, M., Terroux, M., Tremblay, B., Marchese, L., Savard, E., Doucet, M., \& Bergeron, A. (2011). Noise-equivalent power characterization of an uncooled microbolometer-based $\mathrm{THz}$ imaging camera. SPIE Defense, Security, and Sensing, 80230C-80230C.

[11] Oden, J., Meilhan, J., Lalanne-Dera, J., Roux, J. F., Garet, F., Coutaz, J. L., \& Simoens, F. (2013). Imaging of broadband terahertz beams using an array of antenna-coupled microbolometers operating at room temperature. Optics express, 21(4), 4817-4825. 
[12] G. N. Kulipanov, N. G. Gavrilov, B. A. Knyazev, E. I. Kolobanov, V. V. Kotenkov, V. V. Kubarev, A. N. Matveenko, L. E. Medvedev, S. V. Miginsky, L. A. Mironenko, V. K. Ovchar, V. M. Popik, T. V. Salikova, M. A. Scheglov, S. S. Serednyakov, O. A. Shevchenko, A. N. Skrinsky, V. G. Tcheskidov, and N. A. Vinokurov, "Research highlights from the Novosibirsk $400 \mathrm{~W}$ average power THz FEL," Terahertz Sci. Technol. 1(2), 107-125 (2008).

[13] C. Pradere, J.P. Caumes, D. Balageas, S. Salort, E. Abraham, B. Chassagne, J.C. Batsale, (2010), "Photothermal converters for quantitative 2D and 3D real-time TeraHertz imaging", Quantitative InfraRed Thermography, ISSN: 1768-6733, vol 7, n², pp 217-235.

[14] F. Deprettere, SVD and signal processing: algorithms, analysis and applications, Elsevier Science Publishers, Amsterdam, 1988.

[15] B.S. Everitt, and G. Dunn, Applied multivariate data analysis, Arnold, London, 2001.

[16] B.S. Everitt, S. Landau, and M. Leese. 2001. Cluster analysis. Arnold, London, 2001.

[17] Krapez J.-C., Spagnolo L., Frieß M., Maier H.-P., Neuer G.- "Measurement of in-plane diffusivity in nonhomogeneous slabs by applying flash thermography" - International Journal of Thermal Sciences , 43 (2004), pp967-977.

[18] I. Philippi, J.C. Batsale, D. Maillet, A. Degiovanni, "Measurement of thermal diffusivity trough processing of infrared images," Rev. Sci. Instrum, 66[1] 182-192 (1995).

[19] M.W. Berry, S.T. Dumais, and G.W. Obrien, Using linear algebra for intelligent information-retrieval, SIAM Review 37 (1995), pp. 573-595.

[20] E. Palomo del Barrio, An efficient computational method for solving large-scale sensitivity problems, Numerical Heat Transfer Part B 43 (2003), pp. 353-372.

[21] H.M. Park, and W.S. Jung, On the solution of inverse heat transfer using the Karhunen-Loève Galerkin method, Int. J. Heat Mass Transfer 42 (1999), pp. 127-142.

[22] E. Palomo del barrio, J.L. Dauvergne, C. Pradere, (2012), "Thermal characterization of materials using Karhunen-Loève decomposition techniques. Part I. - Orthotropic materials" Inverse Problems in Science and Engineering, ISSN: 1741-5977, vol 20, issue 8, pp. 1115-1143. 